\title{
Du document de genèse à la genèse d'un genre
}

Le carnet d'écrivain dans la littérature française du $\mathrm{xx}^{\mathrm{e}}$ siècle

\section{Sophie Hébert}

\section{(2) OpenEdition}

1 Journals

Édition électronique

URL : http://journals.openedition.org/genesis/1720

DOI : 10.4000/genesis. 1720

ISSN : 2268-1590

\section{Éditeur :}

Presses universitaires de Paris Sorbonne (PUPS), Société internationale de génétique artistique littéraire et scientifique (SIGALES)

\section{Édition imprimée}

Date de publication : 12 décembre 2016

ISBN : 9791023105490

ISSN : 1167-5101

Référence électronique

Sophie Hébert, "Du document de genèse à la genèse d'un genre », Genesis [En ligne], 43 | 2016, mis en ligne le 01 décembre 2017, consulté le 03 mai 2019. URL : http://journals.openedition.org/

genesis/1720 ; DOl : 10.4000/genesis. 1720 


\section{Du document de genèse à la genèse d'un genre Le carnet d'écrivain dans la littérature française du xxe siècle*}

Sophie Hébert

$\mathrm{S}$ i le sacre du carnet prend place au XIX ${ }^{\mathrm{e}}$ siècle, alors que les auteurs, réalistes et naturalistes en particulier, introduisent et généralisent la pratique de ce support dans le cadre de leurs recherches avant-textuelles et plus généralement dans leur travail de création littéraire, c'est au $\mathrm{XX}^{\mathrm{e}}$ siècle que le carnet en tant qu'objet matériel devient progressivement objet d'étude. Les causes de cette étape décisive dans l'histoire du carnet sont multiples, interdépendantes et méritent que l'on s'y attarde d'emblée.

En premier lieu, l'attention théorique et critique portée au carnet est indissociable de la démultiplication, sous l'angle éditorial, des ouvrages génériquement associés à la littérature personnelle : à ce titre, la publication du journal d'André Gide, à partir de 1939, tout autant que l'influence de cet auteur dans la sphère littéraire de son temps, constituent un véritable « choc exogène ${ }^{1}$ », qui fut aussi propice au désir de carnet chez les écrivains non encore convertis à ce support que favorable à l'infléchissement de l'écriture carnétiste dans son association étroite avec le genre du journal. Il est intéressant de remarquer que le phénomène touche également des publications qui échappent au champ spécifiquement littéraire : en effet, les années 1945-1965 sont particulièrement riches en carnets rédigés par des hommes politiques soucieux de faire état de leur expérience des deux guerres mondiales ${ }^{2}$. Il serait possible de citer, en outre, quelques carnets de sociologues, comme Lucien Lévy-Bruhl ${ }^{3}$, ou encore de philosophes comme Emmanuel Mounier ${ }^{4}$ ou Maurice Blondel ${ }^{5}$. Ces exemples ne représentent ici qu'une part infime des publications relatives à l'écriture personnelle : mais ils suffisent à prouver que le terme «carnet», de manière significative, investit, de façon croissante, l'espace du titre dans les œuvres, littéraires ou non, du XXe siècle.

En deuxième lieu, l'avènement de la génétique textuelle et de la critique génétique a incontestablement modifié le rapport de l'écrivain à son carnet, soudain devenu espace d'intérêts, de recherches et d'analyses au même titre que le reste de son œuvre. De fait, et même si quelques cas de publications anthumes sont attestés avant l'apparition de la génétique (on pense ici à un carnet de Jean Guéhenno et d'Henri Thomas, à la majeure partie des carnets de Louis Guilloux mais surtout à la production carnétiste d'Henry de Montherlant), c'est à partir des années 1970-1980 que la publication de ces textes se généralise et s'offre au lecteur dans le souci de lui faire découvrir les coulisses de la création littéraire : on édite des carnets posthumes, afin de faire revivre, selon une approche nouvelle, certains auteurs majeurs de la littérature française du $\mathrm{XX}^{\mathrm{e}}$ siècle (Roland Barthes, Albert Camus, Jean Grenier, André Malraux, Antoine de Saint-Exupéry ou Jean-Paul Sartre) tandis que, de leur côté, les écrivains (Julien Gracq, Philippe Jaccottet)

* Cet article présente quelques réflexions nées à la faveur d'une thèse de doctorat intitulée «Pour une poétique du carnet d'écrivain dans la littérature française du XXe siècle», dirigée par Claude Coste et soutenue en décembre 2014 à l'Université Stendhal-Grenoble III.

1. «L'accident peut prendre différentes formes. La plus nette, la plus typique, mais pas nécessairement la plus fréquente ou la plus significative, est le choc exogène. Un événement extérieur vient infléchir le cours de l'œuvre» (je souligne), dans Daniel Ferrer, Logiques du brouillon. Modèles pour une critique génétique, Paris, Éditions du Seuil, coll. «Poétique», 2011, p. 55.

2. Voici quelques titres: Michel Tony-Révillon, Mes carnets (juinoctobre 1940). Documents et témoignages pour servir à l'histoire, Paris, Odette Lieutier, 1945 ; Pierre Bourdan, Carnet de retour avec la division Leclerc et Carnet des jours d'attente (juin 1940-juin 1944), Paris, Trémois, 1945 ; Pierre Descaves, Le Carnet rouge, Paris, Défense de la France, 1948; André François-Poncet, Carnets d'un captif, Paris, Fayard, 1952; Abel Ferry, Les Carnets secrets (1914-1918), Paris, Grasset, 1957 ; Louis Loucheur, Carnets secrets, Turnhout, Brepols, 1962.

3. Lucien Lévy-Bruhl, Carnets, Paris, Presses universitaires de France, 1949.

4. Emmanuel Mounier, Carnets de route, Paris, Éditions du Seuil, 1950 (t. I), 1951 (t. II), 1953 (t. III).

5. Maurice Blondel, Carnets intimes, Paris, Éditions du Cerf, 1961. 
n'hésitent plus à rendre publics ces textes particuliers, faisant désormais partie intégrante de l'œuvre entier.

En troisième lieu, les coïncidences structurelles, bien que supposées fruits du hasard, entre l'écriture carnétiste et certains intérêts théoriques du deuxième $\mathrm{XX}^{\mathrm{e}}$ siècle ne furent pas étrangères à la poursuite et à la pérennisation de ces publications, de l'attrait des lecteurs pour les écritures privées et notamment pour le genre du journal, à l'attention portée à l'écriture discontinue, en passant par le goût du fragment et de l'écriture notulaire.

Mais la publication de ces carnets d'écrivain est loin de se résumer à une mise en avant éditoriale d'un document de genèse propre à éclairer, de manière rétrospective, une œuvre littéraire. En d'autres termes, le geste de publication semble avoir fait basculer le carnet vers autre chose, qui se situe bien au-delà de la seule réalité matérielle à laquelle il était étroitement rattaché en tant que support. Voici le problème qu'il convient à présent de soulever : comment, au XXe siècle, le carnet est-il passé du statut de support avant-textuel à celui de forme dépositaire d'un imaginaire du support? La thèse peut sembler bien audacieuse : n'est-on pas dans cette «extension du domaine de la littérature» impliquant nécessairement une « dilution du littéraire ${ }^{{ }}$» que dénonce William Marx? Par définition, «si tout devient littérature, plus rien ne l'est», «puisque tout brouillon littéraire, tout fragment, toute paperole peut et doit devenir objet d'étude ${ }^{7} \gg$ : la perte de la valeur textuelle, estimée dans l'ailleurs de l'œuvre de l'auteur, guette ${ }^{8}$, mais il suffit de compulser quelques carnets des auteurs susnommés pour prendre conscience que le carnet publié, au XXe siècle, n'est ni «brouillon ${ }^{9}$ », ni «paperolle», même si l'on conviendra que le passage d'un manuscrit à sa proposition éditoriale se déroule rarement sans heurts ni même manipulations diverses. C'est ici la relation complexe et parfois contradictoire entre le support, le titre et le texte qui explique en grande partie cette métamorphose du carnet qui, de simple document de genèse, devient, au cours du dernier siècle, forme littéraire assumée et prisée, comme le prouvent, de manière complémentaire, l'état des manuscrits (raturés, dactylographiés, échangés même), la généralisation des publications anthumes de carnets mais également l'épitexte auctorial, privé comme public, qui fait mention du carnet non comme un infratexte, mais plutôt comme une ouverture formelle, un nouveau possible générique. De surcroit, n'est-on pas ici dans la mythologie du genre littéraire qui consiste à forcer la catégorisation de textes pourtant mal assortis ? $\mathrm{Si}$ tel est le cas, bien des poéticiens, de Jean-Yves Tadié à Anne Prouteau, en passant par Bernhild Boie ou Michèle Touret 10 tombent, avec moi, dans le piège de cette intuition - qui est aussi ambition - générique, en affirmant que le carnet, tel qu'il se présente chez les auteurs dont ils sont spécialistes, est certainement genre mais certainement pas journal, se construisant même contre lui, cherchant à développer une pratique, une posture et un imaginaire bien distincts de

6. William Marx, «Les résistances théoriques à la critique génétique», dans La Création en acte. Devenir de la critique génétique, dir. Paul Gifford et Marion Schmid, Amsterdam/New York, Rodopi, coll. «Faux Titre/289», 2007, p. 62.

7. Ibid.

8. Michel Contat, «La question de l'auteur», dans Michel Contat (dir.), L'Auteur et le manuscrit, Paris, Presses universitaires de France, 1991, p. 18. «Et ce qui rend en définitive, sinon licite et morale, du moins souhaitable et légitime la publication d'un texte contre la volonté (exprimée ou non) de son auteur, c'est sa valeur. De telle sorte que si la publication du Journal de Barthes a pu choquer, ce n'est peut-être pas tant parce qu'il révélait une homosexualité que l'écrivain n'avait pas voulu assumer du vivant de sa mère, qu'à cause d'une faiblesse manifestée par ce texte à l'endroit d'un régime d'écriture classiquement "auctorial" [...].» Il n'existe pas, à proprement parler, de Journal de Barthes : Michel Contat pense-t-il au carnet intitulé «Incidents »?

9. Hormis, logiquement, les carnets ouvertement génétiques : on pense ici aux Carnets 1, 2, 3, 4 de Marcel Proust, dont l'édition a été établie et présentée par Florence Callu et Antoine Compagnon et publiée chez Gallimard en 2002.

10. «On ne l'imagine pas [Malraux] résumant ses idées, racontant ses sentiments et sa vie intérieure au jour le jour. Il est au contraire entièrement tourné vers le monde extérieur, tout de sensations, d'impressions et d'anecdotes [...]. Ces notes ressemblent à celles des Carnets de Proust, qui a toujours refusé de tenir un Journal », écrit Jean-Yves Tadié dans la préface des Carnets du Front populaire (1935-1936) de Malraux (édition établie et annotée par François de Saint-Cheron, Paris, Gallimard, 2006, p. 7); «les curieux en seront pour leurs frais : ils ne découvriront dans les Carnets de Camus ni détails intimes ni étalage exhibitionniste. L'expérience de soi n'emprunte guère au genre habituel », avance Anne Prouteau à l'entrée «Carnet» du Dictionnaire Albert Camus, dir. Jeanyves Guérin, Paris, Laffont, coll. «Bouquins», 2009, p. 122; pour Bernhild Boie les Lettrines de Gracq «ne se font ni journal ni chronique» (introduction à Julien Gracq, Euvres complètes, édition établie par Bernhild Boie, Paris, Gallimard, coll. «Bibliothèque de la Pléiade», 1995, t. II, p. X-XI). Pour Michèle Touret, «les Carnets de Louis Guilloux ne sont pas un journal : pas de notations au jour le jour mais des réflexions, irrégulièrement inscrites, parfois sans date» : «Louis Guilloux et Jean Paulhan», dans Louis Guilloux écrivain, dir. Francine Dugast-Portes et Marc Gontard, Rennes, Presses universitaires de Rennes, coll. «Interférences », 2000, p. 10. 
ceux des diaristes. Dans la mesure où la «fabrique du genre [du carnet]», pour reprendre l'heureuse expression d'Hervé Ferrage à propos des carnets de Philippe Jaccottet, est une réalité pressentie par un grand nombre de chercheurs s'étant penché sur les carnets du XX $\mathrm{X}^{\mathrm{e}}$ siècle, n'est-il pas nécessaire, du moins légitime, de chercher à harmoniser l'ensemble de ces approches?

Considérer les carnets d'écrivains dans la littérature française du $\mathrm{XX}^{\mathrm{e}}$ siècle impose donc de démêler en priorité de multiples nœuds, théoriques et pratiques, à la lumière vacillante d'un corpus aux formes instables - d'autant plus dangereux qu'il se propose d'emblée aux lecteurs sous une forme publiée. Fallacieuse facilité pour le chercheur, puisque la perspective génétique, à l'origine exclusive, semble devoir s'ouvrir aux questionnements générique et même éditorial. L'idéal serait de posséder un outil efficace permettant de localiser l'ensemble des manuscrits des textes retenus, loin de se situer tous à la Bibliothèque nationale de France ou à la Bibliothèque littéraire Jacques Doucet. «Où trouver des carnets?», principalement manuscrits, est donc aujourd'hui une de mes préoccupations principales - faisant écho, en cela, à l'interrogation de Philippe Lejeune en 201111 : des inventaires précis restent à établir et des bouteilles à la mer, destinées aux collectionneurs privés, à lancer. Imaginons tous les avantages, se déclinant bien au-delà de l'analyse de la relation carnet-œuvre, de ce répertoire : la constitution de séries assez stables pour venir confirmer l'existence d'une poétique du carnet d'écrivain propre à la littérature française du XX $\mathrm{X}^{\mathrm{e}}$ siècle, l'établissement précis d'une histoire de la pratique carnétiste au cours du siècle dernier ou encore l'étude comparée de manuscrits permettant de distinguer clairement, sous l'angle génétique et plus seulement générique, carnets et journaux.

C'est donc l'histoire d'une dématérialisation autant que d'une littérarisation qu'il faut à présent mettre en œuvre, et qui ne peut s'affranchir d'une réflexion théorique préalable sur le carnet en tant que support.

\section{Le carnet n'est pas un cahier}

Carnet, calepin, cahier, bloc-notes, agenda : le vertige terminologique relatif aux supports de poche étourdit autant qu'il effraie. La description matérielle de ces objets permet néanmoins de faire émerger quelques invariants salutaires : le bloc-notes est constitué d'une reliure supérieure, l'agenda est préalablement daté, le calepin présente, par rapport au carnet dont il est matériellement le plus proche, un nombre restreint de feuillets reliés. L'enquête définitoire se resserre donc autour de deux frères ennemis : le carnet et le cahier. Nombreux sont leurs points communs, de leur origine étymologique 12 à la reliure centrale qui les caractérise de façon minimale. Les concernant, seul l'argument du format du support semble opératoire : puisque le calepin est un petit carnet, le carnet serait un petit cahier. Mais ce constat, à la simplicité presque suspecte, engendrerait, pour devenir loi, une nécessité problématique, à savoir l'établissement arbitraire d'un carnet-étalon, aux dimensions fixes - c'est-à-dire fixées -, qui permettrait de placer chaque support concerné dans la catégorie la plus adéquate (carnet ou cahier). Ainsi serait-il possible de répondre à cette interrogation duelle, à ce jour sans réponse claire : où finit le carnet, où commence le cahier?

L'établissement d'une loi, qui serait ici limite formelle, semble aussi pratique qu'idéale : telles dimensions, en longueur ou en largeur, marqueraient l'avènement du cahier et l'exclusion définitive du carnet - et inversement. Pourquoi ne pas supposer que cette loi existe déjà, tacitement, chez les fabricants de ce type de supports? Le site Internet de Moleskine $®$, marque légendaire de légendaires carnets, propose deux paradigmes matériels spécifiques, l'un pour le carnet, l'autre pour le cahier. Mais cette distinction se révèle, en dépit de tout, infructueuse : en quelques années, les dimensions des supports Moleskine ${ }^{\circledR}$ ont varié (voir encadré page suivante), preuve de l'insaisissabilité de l'objet même en termes marketing. Quelles peuvent être les raisons de telles modifications? Réclamations répétées de clients fidèles mais insatisfaits, chaudes recommandations d'universitaires ou d'aventuriers? Le mystère reste entier. Mais la distinction du carnet et du cahier par le seul argument de la taille du support se révèle donc, dans les faits, rigoureusement aporétique, et l'on peut ajouter que de toutes les façons cette division stricte aurait détruit, à terme, le dynamisme intrinsèque de ces objets, en figeant définitivement leurs contours.

11. Philippe Lejeune, «Où trouver des journaux ? », Genesis, n 32, «Journaux personnels», 2011, p. 190-192.

12. Carnet et cahier viennent du latin «quaternio», soit «cahier de quatre feuillets de quatre pages» pour reprendre la définition exacte de Félix Gaffiot. 


\section{MOLESKINE}

En 2010, carnet et cahier étaient proposés chacun dans trois formats différents : un «petit format» $(9 \mathrm{~cm} \times 14 \mathrm{~cm})$, un «grand format» $(12 \mathrm{~cm} \times 21 \mathrm{~cm})$ et un «très grand format» $(19 \mathrm{~cm} \times 25 \mathrm{~cm})$. En 2016, carnet et cahier sont disponibles, cette fois-ci, en quatre formats différents, mais seul le «très grand format» $(19 \mathrm{~cm} \times 25 \mathrm{~cm})$ reste inchangé. En effet, alors que le «grand format» version 2010 perd seulement 0,5 centimètre en largeur $(11,5 \mathrm{~cm} \times 21 \mathrm{~cm})$ dans sa version 2016 , ce qui était «petit format» en 2010 devient «moyen format» en 2016, avec une modification significative de la hauteur du support (qui passe de 14 à $18 \mathrm{~cm}$ ). Enfin, incontestable nouveauté, naît la catégorie «format de poche», qui conserve la hauteur du «petit format» version 2010, mais voit sa largeur diminuer de $1,5 \mathrm{~cm}$.

Cependant, cette approche n'est pas à abandonner totalement, car elle gagne en pertinence lorsqu'elle s'augmente d'une autre perspective théorique, qui consiste à identifier les supports autant en regard de leur réalité matérielle que de l'utilisation qu'ils supposent de la part des écrivains - en somme peut-être faudrait-il s'intéresser moins à la dimension qu'à la fonction. C'est d'ailleurs le point de vue adopté par Louis Hay en 1990 lorsque, dans un article intitulé «L'amont de l'écriture 13 », le généticien fait du cahier un support sédentaire («de table») et du carnet un support mobile («de poche $14_{»)}$ : mais à distinction nette, conséquences complexes. En premier lieu, l'hypothèse du format du support ne doit pas être complètement écartée : pour être transporté, le carnet doit revêtir des dimensions raisonnables - la tablette latine, ancêtre fantasmé du carnet, ne se nommait-elle pas «pugillares», qui tient dans le poing 15 ? En deuxième lieu, dans la mesure où la dimension du support détermine un régime d'écriture spécifique ${ }^{16}$, carnet et cahier doivent développer des contenus manuscrits distincts - quitte à avancer, grâce aux hypothèses d'Almuth Grésillon, que, dans certains cas, carnet et cahier ne s'utilisent pas, dans le temps de la création, au même moment ${ }^{17}$. En troisième lieu, si le cahier est un support sur lequel on écrit à l'intérieur et le carnet à l'extérieur, cela suppose que des imaginaires d'écriture différents soient à l'œuvre : dès lors, une opposition à la fois physique et métaphorique entre le dedans et le dehors semble possible et même recevable sous l'angle théorique.

La théorie se complique enfin définitivement : au-delà du fait que le carnet n'est pas l'unique matériau de travail de l'écrivain, qui peut tout à fait manipuler en une même journée plusieurs supports différents (le carnet pour les idées nées à l'extérieur, le cahier pour la tenue d'un journal personnel, par exemple), quelques cas pratiques, incarnés par Roland Dubillard ou Antoine de Saint-Exupéry, viennent démontrer que les auteurs utilisent parfois plusieurs carnets en même temps :

En effet, si Roland Dubillard accorde une grande importance à l'exactitude des dates, il a tenu à ce que chaque carnet conserve son intégrité et a refusé de sacrifier celle-ci à un ordre chronologique qui aurait impliqué une dissection des carnets. Or, ces derniers, commencés, abandonnés puis repris parfois plus de dix ans après, et délaissés à nouveau, se chevauchent dans leur succession. De même, à l'intérieur d'un simple carnet, il est arrivé que l'auteur revienne sur des pages antérieurement rédigées ou restées blanches, pour $\mathrm{y}$ ajouter des notes avant de reprendre son cahier où il l'avait

13. Louis Hay, «L'amont de l'écriture», dans Carnets d'écrivains 1 - Hugo, Flaubert, Proust, Valéry, Gide, du Bouchet, Perec, dir. Louis Hay, Paris, Éditions du CNRS, coll. «Textes et manuscrits», 1990.

14. Autrement dit, tout carnet serait, par nature, «de poche» ce qui vient, de fait, affaiblir la pertinence de la création d'un «format de poche» chez Moleskine ${ }^{\circledR}$.

15. Richard H. Rouse et Mary A. Rouse, «Sept siècles de littérature manuscrite», dans La Naissance du texte, dir. Louis Hay, Paris, José Corti, 1989 p. 89-103. Les pages 90 à 94 sont particulièrement consacrées à la tablette de cire.

16. «[Ce] qui distingue ces deux régimes d'écriture [carnet et cahier] serait précisément le support matériel. Portatif dans le cas du carnet, il favorise la prise de notes courtes ou des unités textuelles plutôt brèves, rédigées en voyage, au café, en promenade. Moins amovibles dans le cas du cahier, le support conduit à la constitution d'unités textuelles longues et qui peuvent être reprises d'une séance de travail à l'autre. Ainsi le cahier implique plutôt un travail sédentaire, le carnet une écriture nomade», dans Michel Contat et Jacques Deguy, «Les Carnets de la drôle de guerre de Jean-Paul Sartre : effets d'écriture, effets de lecture», Littérature, $\mathrm{n}^{\circ} 80$, «Carnets, Cahiers», décembre 1990, p. 19.

17. Almuth Grésillon, La Mise en æuuvre. Itinéraires génétiques, Paris, CNRS Éditions, 2008, p. 56. 
laissé. Il est probable également qu'il a recopié des passages écrits antérieurement sur des feuilles séparées, ce qui expliquerait la succession des dates pour le moins étonnante de plusieurs carnets 18 .

[Saint-Exupéry] se servait parfois de plusieurs carnets en même temps selon qu'il oubliait, égarait l'un et en avait un autre sous la main. Par ailleurs, pour le contenu même, il arrive à sa pensée de remonter librement, dans tel ou tel fragment, vers un passé plus ou moins lointain, rendant parfois hasardeuse la restitution d'un ordre chronologique 19.

Dans les deux cas, la logique d'emploi semble plutôt chaotique car soumise au hasard. Seul l'argument de la spatialisation semble opératoire : j'écris sur le carnet le plus proche de moi afin d'éviter l'oubli qui guette toujours plus ou moins l'idée qui vient de naître en moi - ce qui explique que le carnet doive toujours être à portée de main, d'où la nécessité de la remise en question du rapprochement entre carnet et journal, étant donné l'indifférence de nombreux carnétistes pour la chronologie. Un manque ici apparaît, relatif à la description matérielle des carnets : quid d'une potentielle fonctionnalisation des supports? Le carnet rouge, par exemple, serait mon carnet de notes de lectures, tandis que le carnet bleu serait le support préparatoire noirci en vue de mon prochain roman.

Ainsi le carnet répond-il à une double dimension paradoxale : spatiale (dans sa pratique en extérieur et dans sa proximité avec son possesseur) autant qu'atemporelle (certains auteurs étant animés par une logique de collection qui semble pouvoir faire fi de toute idée de succession).

\section{Les trois carnets de Louis Hay}

Sous l'angle génétique, le cas d'école est le suivant : le carnet occupe une partie de l'avant-texte et se présente comme un espace préparatoire riche d'hésitations, de bifurcations et d'accidents. Autrement dit, le carnet se définit, de façon rudimentaire, à partir de ces trois traits : la dimension, la fonction, l'inscription. Louis Hay, à qui le carnet doit beaucoup sous l'angle théorique, a proposé une typologie discernant le «carnet d'esquisses», qui est «celui des premiers instantanés textuels : vers, idées, expressions, [sont] épinglés sur le coup pour ne pas échapper à la mémoire ${ }^{20}$ » et le «carnet d'enquête»:
Ce sont toujours des supports mobiles où s'inscrivent des notes rapides, mais destinées cette fois à une œuvre déjà en route, ou du moins à un projet d'écriture. Leur matière première, c'est la documentation; leur terrain, celui des bibliothèques et archives, mais aussi des repérages sur le motif; leur champ, la société contemporaine autant que l'histoire; leur source d'information, des documents, des faits, des témoignages $-\mathrm{le}$ «lu, le vu, l'entendu ». [...] L'analyse de tels documents est rendue difficile, ne serait-ce que par leur volume [...] mais aussi par le brouillage de leur statut génétique : entre l'information et l'invention, entre le documentaire et l'imaginaire 21.

Le carnet dit «composite», quant à lui, trouble profondément les définitions des deux premiers types en même temps qu'il peine à caractériser sa propre matière. S'il est qualifié de «composite», c'est qu'il contient, en regard du «carnet d'esquisses » et du «carnet d'enquête», une materia prima plus hétérogène (esquisse sténographique née d'une chose vue, entendue ou pensée, premier jet fictionnel, note de lecture, plan d'ouvrages à venir, trace du quotidien voire biographème) et fait également l'objet d'une pratique moins saisissable, plus erratique, de la part de l'auteur :

L'imprécision du terme semble décourager d'avance toute ambition typologique [...]. Objet et fonction se rejoignent ici pour créer le lieu privilégié d'une pratique de l'écriture qui enregistre pêle-mêle l'éphémère et l'essentiel, événements quotidiens et projets littéraires, fragments de formes ou d'idées. Le carnet participe ainsi à une étape bien particulière - nullement obligatoire, d'ailleurs - de la genèse. [...] Tout cela offre un merveilleux observatoire sur l'univers quotidien de l'écrivain, ses sensations premières, ses réactions crayon en main 22 .

Si le «carnet d'enquête», catégorie que Louis Hay emprunte à Henri Mitterand et qui est sans doute parasynonyme de celle de «carnet de travail» portée par Pierre-Marc de Biasi,

18. Diane Henneton, dans Roland Dubillard, Carnets en marge, préface de Robin Wilkinson, avant-propos, notes et chronologie de Diane Henneton, Paris, Gallimard, 1998, p. X-XI

19. Michel Autrand, à propos des Carnets de Saint-Exupéry, dans Antoine de Saint-Exupéry, Euvres complètes, dir. Michel Autrand et Michel Quesnel, Paris, Gallimard, coll. «Bibliothèque de la Pléiade», 1994, t. I, p. 1122.

20. Louis Hay, «L'amont de l'écriture», art. cité, p. 10. Je souligne.

21. Ibid., p. 11. Je souligne.

22. Ibid., p. 12. Je souligne. 
semble, dans cette typologie, le plus facilement conceptualisable, c'est qu'il est aujourd'hui le mieux défini car le plus étudié, porté par la génétique textuelle et la critique génétique, illustré par quelques ouvrages canoniques, à l'image des deux éditions critique et génétique des carnets zoliens ou flaubertiens à la fin des années $1980^{23}$. En revanche, la distinction entre «carnet d'esquisses » et «carnet composite» manque quelque peu d'évidence : si l'on suit la logique de Louis Hay, «carnet d'esquisses » et «carnet composite» sont tous deux des recueils d'idées premières. La solution théorique pourrait alors être la suivante : la définition du «carnet d'esquisses » selon Louis Hay est assez générale pour convenir à tout carnet, puisque s'y déposent librement toutes manifestations de pensées estimées valables, jugées remarquables, dignes d'être saisies et, pourquoi pas, retravaillées puis diffusées. Dans ce cas, la définition du «carnet d'esquisses » permettrait d'offrir une définition historique de la forme «carnet» : tout carnet serait de poche, tout carnet serait lieu d'esquisses.

Reste le «carnet composite», dont la réticence à être défini est, avouons-le, stimulante. S'il répond, à l'instar du «carnet d'esquisses » et du «carnet d'enquête», à une «poétique de l'urgence»- puisque l'auteur, redoutant la disparition de ce qu'il vient à l'instant de penser, de voir ou de sentir, écrit pour contrer l'oubli -, autre chose en lui doit être à l'œuvre qui n'est pas sous le joug exclusif de la naissance - naissance de l'idée, comme pour le «carnet d'esquisses », naissance du texte, comme pour le «carnet d'enquête». Le «carnet composite» n'est donc pas essentiellement un strict document premier, préparatoire, puisqu'il se nourrit de quelque chose qui dépasse, voire échappe - cherche à échapper ? - au processus de la genèse créatrice. Pour résumer, si ce type de carnet se présente comme «composite», c'est qu'il n'est pas le support exclusif des commencements. Le problème reste que les carnets envisagés sont des carnets d'écrivains, ce qui a deux conséquences non négligeables dans l'analyse. D'abord, en soi, toute note peut devenir préparatoire à partir du moment où le scripteur a pour métier la littérature, ce qui fait de tout «carnet composite» un «carnet d'enquête» en puissance : en effet, l'esquisse sténographique peut devenir fragment prérédactionnel, la note de lecture prétexte à essai, le biographème élément clé de fiction - le passage de l'un à l'autre étant aussi ténu qu'aléatoire puisque soumis aux aléas capricieux de l'inspiration créatrice. Ensuite, la période considérée étant le $\mathrm{XX}^{\mathrm{e}}$ siècle, un regard soupçonneux sur tout ce qui se dit genèse dans un espace publié est en droit d'être jeté : pourquoi ne pas imaginer un exercice de style qui consisterait à présenter un produit en le faisant passer pour un processus? Dans ce cas, le «carnet composite» serait donc la proposition textuelle la plus aboutie des trois théorisées par Louis Hay. Cette conséquence théorique est aussi surprenante qu'intéressante puisque, à première vue, le «carnet composite» semblait justement celui qui, dans sa pratique, était le moins réfléchi, faisant figure de libre réceptacle capable de rassembler ce que la pensée peut avoir de plus désordonné.

Le «carnet composite» est donc la catégorie qui convient le mieux pour réunir, de manière souple cependant, l'ensemble des carnets d'écrivain qui, au $\mathrm{XX}^{\mathrm{e}}$ siècle, ne sont pas exclusivement premiers («carnet d'esquisses») ou préparatoires («carnet d'enquête») et font l'objet d'une pratique scripturale spécifique. Cet ensemble d'hypothèses, qui cherche de manière générale à déstabiliser l'idée selon laquelle tout carnet serait préparatoire, c'est-à-dire au service d'une œuvre littéraire à venir, dit également quelque chose de la relation auteur-genèse, décisive au $\mathrm{XX}^{\mathrm{e}}$ siècle, et en particulier depuis l'institutionnalisation de l'étude des manuscrits littéraires. Le regard du lecteur, du critique et de l'éditeur porte désormais autant sur le processus littéraire que sur le produit : on attend que soient dévoilés, pour reprendre quelques clichés du genre, les coulisses de l'œuvre, la fabrique du texte, le laboratoire de l'écrivain. Ce changement de cap critique est loin d'avoir échappé aux écrivains, et de cette prise de conscience de la valeur de la genèse littéraire découlent naturellement plusieurs attitudes auctoriales : la destruction du brouillon (l'auteur force le regard extérieur à se concentrer sur le produit, comme le voudrait Louis Guilloux ${ }^{24}$ ), la disparition du brouillon

23. Émile Zola, Carnets d'enquêtes : une ethnographie inédite de la France, textes présentés et établis par Henri Mitterand, Paris, Plon, 1986 et Gustave Flaubert, Carnets de travail, édition critique et génétique établie par Pierre-Marc de Biasi, Paris, Balland, 1988.

24. Geste très régulièrement envisagé, pouvant même être considéré comme une topique de l'écriture carnétiste, mais peu réalisé : «Il me vient des rages silencieuses de tout mettre au feu. Il faudrait avoir ce courage pourtant bien raisonnable de détruire», dans Louis Guilloux, Carnets I, Paris, Gallimard, 1978, p. 238. 
(l'auteur dissimule le processus textuel pour le soustraire au regard critique ou le confie à un ami proche 25$)$, la manipulation du brouillon (l'auteur cherche à influencer le regard extérieur en faisant un faux, comme le fait régulièrement Montherlant en réécrivant dans ses carnets des notes de ses essais, par exemple) ou, enfin, l'usage du «brouillon mental», par définition impossible à prouver matériellement, lors duquel l'auteur dissocie sciemment processus littéraire et trace écrite, faisant se rejoindre premier jet et produit fini, puisque l'usage répété du «brouillon mental»-notion dont on sait qu'elle est pertinente pour des supports proches du carnet, comme les Cahiers de Valéry 26 - viserait, idéalement, à produire une note immédiatement juste et littéraire et pourrait venir justifier l'absence relative de ratures sur le manuscrit tout en expliquant l'impression d'aboutissement textuel à la lecture de certaines notes.

Ainsi, en dépit de l'hétérogénéité de sa matière, le «carnet composite» occupe-t-il une place spécifique dans la conscience génétique de l'auteur et, par conséquent, dans sa pratique scripturale. Dès lors, quelle(s) relation(s) le «carnet composite» entretient-il avec l'œuvre littéraire, et comment passe-t-on du carnet supposé de genèse à la genèse supposée d'un genre?

\section{Du carnet manuscrit au livre publié : «la fabrique du genre» (Hervé Ferrage)}

Reconstituer l'univers intellectuel et créatif de l'écrivain, esquisser ce que Jean Bellemin-Noël nomme «la psychologie de la création littéraire», et ce à partir de conjectures essentiellement fondées sur des discours multiples et parfois contradictoires sur le carnet, est un premier pas risqué, mais, faute de mieux, incontournable. Le recours au carnet publié, dont la situation textuelle, figée et finale, se trouve naturellement aux antipodes de la genèse, fluctuante et féconde, n'est pas sans intérêt cependant, puisqu'il permet, d'ores et déjà, d'épingler des bizarreries qui ne pourront prendre tout leur sens que lorsqu'il sera possible de mettre en regard manuscrit et texte publié. Car l'approche génétique, qui semblait s'épuiser faute de manuscrits à compulser, ne quitte pas pour autant le devant de la scène théorique, prenant la forme, cette fois-ci, ou du paradoxe ou de l'aberration. Voici, modélisés, quatre constats, nés des informations données par le péritexte éditorial et tirés de l'analyse comparée entre le support manuscrit supposé, le titre donné et le texte lu, à partir d'un corpus de «carnets », emprunté à la littérature française du XXe siècle 27 :

Configuration 1 - l'ouvrage a été écrit sur un carnet (support) et porte le nom de carnet (titre choisi par l'auteur ou l'éditeur).

- Cas 1 : le texte appartient au genre du journal. Par exemple, les Carnets (1914-1917) de Jacques Rivière, les Carnets de la drôle de guerre de Jean-Paul Sartre, les Carnets 1944-1971 de Jean Grenier, les deux tomes de Carnets de Louis Guilloux, le Carnet d'URSS de Malraux, les Carnets en marge de Roland Dubillard;

- Cas 2 : le texte n'appartient pas au genre du journal. Par exemple, les Carnets du voyage en Chine de Roland Barthes, les Carnets du grand chemin de Julien Gracq, les Carnets 1930-1944 d'Henry de Montherlant, les Carnets de Saint-Exupéry.

Configuration 2 - l'ouvrage a été écrit sur un carnet (support) mais où la mention «carnet» n'est pas dans le titre, ou elle se trouve reléguée dans un sous-titre (choisi par l'auteur ou l'éditeur).

- Cas 1 : le texte appartient au genre du journal. Par exemple, le Journal intime d'Eugène Dabit, ou encore le De profundis Americae (Carnets américains, 1958-1960) d'Henri Thomas;

- Cas 2 : le texte n'appartient pas au genre du journal. Par exemple, Incidents, de Roland Barthes, Au jour le jour (carnets 1985) de Michel Butor et trois carnets de Montherlant : Va jouer avec cette poussière (carnets 1958-1964), La Marée du soir (carnets 1968-1971), Tous Feux éteints (carnets 1965, 1966, 1967, carnets sans dates et 1972).

Configuration 3-l'ouvrage n'a pas été écrit sur un carnet (support) mais se nomme carnet (titre ou sous-titre choisi

25. Voir la préface de Joanna Leary dans Henri Thomas, Carnets inédits 1947, 1950, 1951 suivi de Pages 1934-1948, dir. Joanna Leary, Paris, Gallimard, coll. «Les Cahiers de la NRF», 2006, p. 9-15.

26. Voir l'annexe intitulée «Le brouillon valéryen», dans Robert Pickering (dir.), Paul Valéry : se faire et se refaire. Lecture génétique d'un cahier (1943), Clermont-Ferrand, Presses universitaires Blaise Pascal, coll. «Cahiers de Recherches du CRLMC», 1996, p. 204-206.

27. Ce classement a été établi à partir d'un échantillon d'une trentaine de carnets, fruit d'un choix subjectif, mais qui se veut représentatif. 
par l'auteur ou l'éditeur). Un seul cas, pour l'instant, a été répertorié, celui où le carnet n'appartient pas au genre du journal. Par exemple, les trois tomes de Carnets d'Albert Camus, le Carnet du Front populaire de Malraux, les Carnets $d u$ vieil écrivain de Jean Guéhenno, les Carnets du grand chemin de Julien Gracq, mais également les trois tomes de Semaison de Philippe Jaccottet, écrits sur des cahiers et sous-titrés ou titrés «carnets ».

Configuration 4-l'ouvrage n'a pas été écrit sur un carnet (support) et ne se nomme pas carnet (titre choisi par l'auteur ou l'éditeur) mais a tout, textuellement, d'un carnet. Par exemple, le Journal du dehors d'Annie Ernaux ou encore les Lettrines de Julien Gracq.

Si ces quatre modèles présentent l'avantage de permettre un agencement minimal d'un corpus dont les frontières sont nécessairement floues, ils n'en sont pas moins problématiques sous certains aspects - hormis la configuration 1 qui symbolise la version idéale de la relation entre le support, le titre et le texte. La configuration 2 est à première vue la plus proche de la configuration 1 , puisqu'elles possèdent la même source génétique : pourtant, sous l'angle textuel précisément, elles sont généralement très différentes - de fait, deux subdivisions ont semblé nécessaires pour rendre compte de la complexité de cette configuration. En effet, dans la plupart des cas liés à ce modèle, le titre ne fait pas référence à un support, mais à un genre qui, dans la majorité des cas, se trouve être le journal (cas 1). Il arrive parfois, cependant, que le titre ne fasse pas référence au genre diariste, mais à autre chose qui n'a pas encore été défini (cas 2). Dans le cas de la configuration 3, l'étrangeté se trouve, une fois encore, dans l'inadéquation entre le titre et le support concerné28. Enfin, la configuration 4 n'a pas, a priori, de raison d'être, puisque le carnet en est absent : pourtant, tout mène, textuellement, vers la forme carnet.

Deux ensembles se dessinent donc : d'une part, les configurations 1 et 2, qui ont les carnets pour origine génétique, d'autre part, les configurations 3 et 4 , qui conservent, dans leur titre ou leur texte, quelque chose du carnet sans que ce dernier soit leur source génétique. Comment expliquer cette dématérialisation à l'œuvre entre les modèles 1 et 2 , d'une part, et les configurations 3 et 4 , d'autre part? Comment comprendre ces diverses tensions entre le support et le titre, qu'elles aient pour origine l'auteur ou l'éditeur critique? Quel horizon d'attente le terme «carnet» informe-t-il pour chercher à occuper la place du titre au mépris parfois de la genèse du texte ou de sa catégorie générique? Sans doute faudrait-il revenir ici à notre analyse de départ sur le support carnet : la pratique qu'il favorise informe un imaginaire qui le dépasse largement - autrement dit, un imaginaire qui peut, in fine, se passer de lui -, et qui touche à la façon de faire de la littérature autant qu'à l'image de l'auteur.

Dans son ouvrage sur les carnets de lecture, Andrei Minzetanu affirme que le carnet est autant objet matériel que pure construction littéraire, travaillée par une posture auctoriale puissante 29 : le carnétiste, en effet, semble à la recherche constante de la «concrétisation d'une rupture [...] qui consiste à sortir des sentiers battus ${ }^{30} \gg$. L'approche sociopoétique peut venir corroborer les hypothèses sur le carnet comme genre, puisque ce type de texte joue un rôle capital dans la paratopie recherchée par l'écrivain «dont l'énonciation se constitue à travers l'impossibilité même de s'assigner une véritable "place". [...] L'existence sociale de la littérature suppose à la fois l'impossibilité de se clore sur soi et celle de se confondre avec la société "ordinaire", la nécessité de jouer de et dans cet entre-deux 31 ». Outre le fait que la métaphore de l'espace générée naturellement par l'idée de «champ (littéraire)» renforce son lien avec le carnet, nomade et marginal, et dans la mesure où la marche, attitude privilégiée du carnétiste, est déjà une «manière de prendre position face à la société 32 » et «un lieu de l'écart 33 », le carnet se révèle l'instrument clé de cette paratopie, cherchant à singulariser son positionnement par rapport au système génériquement stable de l'œuvre et en regard de la production opérale des autres auteurs du moment. S' «il y a probablement à toutes les époques des réseaux d'esprit

28. Les Carnets de Camus, par exemple, se sont intitulés «carnets » sous l'impulsion des éditeurs critiques, afin que ces textes se distinguent nettement des «Cahiers Albert Camus » publiés chez Gallimard : or, les Carnets de Camus ont été écrits sur des cahiers.

29. Andrei Minzetanu, Carnets de lecture : généalogie d'une pratique littéraire, Paris, Presses universitaires de Vincennes, coll. «Manuscrits modernes », 2016

30. Gérard Cogez, Les Écrivains voyageurs au XXe siècle, Paris, Éditions du Seuil, coll. «Points Essais», 2004, p. 26.

31. Dominique Maingueneau, Le Contexte de l'œuvre littéraire : énonciation, écrivain, société, Paris, Bordas, 1993, p. 27-28.

32. Alain Montandon, Sociopoétique de la promenade, Clermont-Ferrand, Presses universitaires Blaise Pascal, coll. «Littératures », 2000, p. 10.

33. Ibid. 
très proches les uns des autres, même s'ils s'ignorent le plus souvent : constellations merveilleuses qu'on aime à redécouvrir dans son propre $\operatorname{ciel}^{34} »$, il faut rappeler que les carnétistes au XXe siècle ne s'ignorent pas, et peut-être est-ce là l'argument le plus sûr pour étayer l'hypothèse selon laquelle le carnet comme nom de genre naît au XXe siècle. Ces affinités entre auteurs, ces parentés textuelles se manifestent de multiples manières car, contrairement au genre du journal, les carnets ne s'écrivent pas à l'insu les uns des autres - conséquence de la pratique publique de cette écriture qui reste néanmoins privée. Sartre, par exemple, dans ses Carnets de la drôle de guerre, remarque la «pompe» avec laquelle Gide choisit ses carnets 35 ; plus généralement les carnétistes se lisent entre eux : Camus, par exemple, cite dans ses carnets ceux de Montherlant qui viennent d'être publiés. De plus, ces textes circulent parfois sous une forme dactylographiée : Camus donne une copie de ses carnets à Guilloux qui, dans une sorte de potlatch, fait de même avec ses propres notes, geste qu'il mentionne, cela semble aller de soi, dans ses carnets. Enfin, les carnétistes éditent des carnétistes : Camus, qui semble donc le centre dynamique de cette famille d'écrivains, donne à Michel Gallimard, dans une lettre datée du 6 décembre 1952, des «conseils quant à l'édition, posthume, des Carnets d'Antoine de Saint-Exupéry $36_{»}$. Ainsi la fondation d'un paradigme générique concernant le carnet peut-elle s'appuyer non seulement sur des textes précis mais encore sur une famille d'auteurs consciente d'elle-même : le corpus, qui semblait à l'origine noyau dur hypothétique, trouve ici cohérence interne et résistance critique, au service d'une certaine stabilité théorique et analytique.
Cherchant à brouiller un certain nombre de clivages théoriques et génétiques en particulier (avant-texte/texte, notamment), remettant en question certaines assimilations génériques (journal/carnet, notamment) pour s'autonomiser définitivement, développant un imaginaire cohérent et dense, favorisant une pratique garantissant en elle-même l'existence d'une véritable famille de carnétistes, les carnets d'écrivains dans la littérature française du XXe siècle peuvent, à bien des aspects, être considérés comme appartenant à un genre propre, indépendant, sous le signe du jeu sur les catégories mais, plus encore, de la liberté scripturale. Recueillant à vau-l'eau, au mépris du temps mécanique et de son inscription régulière, les miroitements variés nés de l'instant primordial d'un «climat mental ${ }^{37}$ », le carnet est détenteur, à l'instar de toute œuvre littéraire, d'une vision spécifique du monde et de l'homme en même temps qu'il est révélateur d'un style de vie.

34. Philippe Jaccottet, La Seconde Semaison. Carnets 1980-1994, Paris, Gallimard, 1996, p. 224.

35. Jean-Paul Sartre, Carnets de la drôle de guerre, nouvelle édition augmentée d'un carnet inédit, texte établi et annoté par Arlette ElkaïmSartre, Paris, Gallimard, 1995, p. 289.

36. «Du bon ordre d'une publication posthume, ou Camus lecteur de Saint-Exupéry », La Lettre de la Pléiade, n 24, avril-mai 2006, p. 2. Lettre lisible en ligne, à l'URL suivante : < www.la-pleiade.fr/La-vie-dela-Pleiade/L-histoire-de-la-Pleiade/Du-bon-ordre-d-une-publicationposthume >. Consultation le 7 avril 2016.

37. Formule heureuse de Robert André, dans «Pages de carnets», La Nouvelle Revue française, $\mathrm{n}^{\circ} 274$, «Journaux intimes et carnets», 1975 , p. 259 : «Plus tard, je me suis remis à noter sur des calepins ce qui me passait par la tête, moins des pensées que l'expression immédiate d'un climat mental.» 
Ancienne élève de l'ENS (Lyon, 2003), docteure en littérature française depuis 2014 ( Pour une poétique du carnet dans la littérature française du XXe siècle», sous la direction de Claude Coste, université Stendhal), professeure agrégée dans l'enseignement secondaire depuis 2011, SOPHIE HÉBERT travaille sur les dimensions génétique et générique du carnet, mais également sur la mise en édition du texte carnétiste. Elle a publié, à ce jour, une dizaine d'articles portant sur Roland Barthes, Albert Camus, Jean Guéhenno, Louis Guilloux ou encore Henry de Montherlant. Elle y interroge les dimensions génériques, posturales, thématiques ou encore discursives de ces textes.

sophie.clementine.hebert@gmail.com

\section{Du document de genèse à la genèse d'un genre}

Le carnet d'écrivain, au Xxe siècle, semble être l'objet d'une mutation radicale née de la confluence fortuite de nombreuses tendances théoriques, génériques comme génétiques. En effet, au fil des publications, fruits de choix auctoriaux ou éditoriaux, le carnet passe du statut de document de genèse, premier mais secondaire, à celui de genre émergent, marginal mais digne d'intérêt, jouant avec la fixité des jalons théoriques, cherchant à libérer le texte autant que l'auteur du carcan des catégories. Par la pratique scripturale qu'il favorise, par l'image de la littérature qu'il véhicule, par la posture auctoriale qu'il facilite, le carnet au XXe siècle se construit, ouvertement, en opposition avec le genre diariste, développant des imaginaires spécifiques, aux incidences textuelles autant que sociopoétiques évidentes.

During the 20th Century, the writer's notebook seems to have undergone a radical mutation born of the fortuitous confluence of numerous theoretical tendencies, both generic and genetic. In fact, with the passing publications, the result of authorial and editorial choices, the notebook goes from being a medium of genesis, primary but of lesser importance, to being an emergent genre, marginal, yet worthy of interest, playing with the fixity of theoretical references, seeking to liberate the text, as much as the author, from the constraint of categories. Through the scriptural practice it encourages, through the image of literature it conveys, through the authorial posture it facilitates, in the 20th Century the notebook grows, openly and in opposition with the diarist genre, developing specific imaginaries which have very clear textual and sociopolitical incidences.

Das Notizbuch des Schriftstellers im 20. Jahrhundert scheint das Objekt eines radikalen Wandels zu sein, der durch das Zusammentreffen von mehreren theoretischen, generischen wie genetischen Tendenzen verursacht wurde. Denn mit Blick auf die Publikationen, welche das Ergebnis der Wahl des Autors und Herausgebers sind, verändert das Notizbuch seinen Status: vom Dokument der Genese - ursprünglich, aber sekundär in der Bedeutung - in Richtung eines hervortretenden eigenen Genres, das trotz seiner Randstellung doch beachtenswert ist, indem es mit der Fixiertheit der theoretischen Referenzen spielt, indem es versucht, den Text wie den Autor von der drückenden Last der Kategorien zu befreien. Durch die schriftstellerische Praxis, die es bevorzugt, durch das Bild der Literatur, welches es vermittelt, durch die Haltung des Autors, welche es ermöglicht, stellt sich das Notizbuch im 20. Jahrhundert offen in einen Gegensatz zum Genre des Tagebuchs, indem es spezifische Vorstellungswelten prägt, die sehr klare textliche und sozio-politische Einflüsse entwickeln.
Durante el siglo XX, la libreta de escritor ha sido objeto de una mutación aparentemente radical, cuyo origen se encuentra en la confluencia fortuita de numerosas tendencias teóricas, genéricas y aun genéticas. En efecto, como consecuencia de su publicación -emprendida por el escritor o por el editor- la libreta ha pasado de la categoría de documento de génesis (inaugural, pero secundario) a la de género emergente (marginal, pero digno de interés), que juega con la rigidez de los jalones teóricos, tratando de liberar al texto y al autor del yugo de las categorías. Gracias a la práctica escritural que favorece, a la imagen de la literatura que conlleva, a la postura autoral que facilita, la libreta del siglo XX se construye en oposición abierta con el género diarista, desarrollando imaginarios específicos, con incidencias tanto textuales como sociopoéticas evidentes.

$\mathbf{O}$ caderno de notas do escritor, no século XX, parece ser o objeto de uma radical mutação, nascida da confluência fortuita de muitas tendências teóricas, tanto de género como de génese. Com efeito, ao sabor de publicações que são fruto de opções de autor ou de editor, o caderno passa do estatuto de documento genético, primitivo mas secundário, ao de género emergente, marginal mas interessante, por jogar com a rigidez das balizas teóricas para libertar o texto e o autor de categorias impositivas. Com a prática de escrita que promove, com a imagem da literatura que veicula, com a postura autoral que facilita, o caderno do século XX é construído, abertamente, em oposição ao tipo do diário e desenvolve imaginários específicos, com implicações textuais e sociopoéticas evidentes.

Nel XX secolo il carnet dello scrittore sembra essere l'oggetto di una mutazione radicale nata dalla confluenza fortuita di numerose tendenze teoriche sia riguardo ai generi, sia alla genetica. In effetti, con il procedere delle pubblicazioni, frutto di scelte autoriali o editoriali, il carnet passa dallo statuto di documento genetico, cronologicamente primo ma d'importanza secondaria, a quello di genere emergente, marginale ma degno d'interesse, che gioca con la fissità dei punti di riferimento teorici, cercando di liberare testo e autore dalle costrizioni delle categorie. Per la pratica scritturale che favorisce, per l'immagine della letteratura che veicola, per la postura autoriale che facilita, il carnet nel XX secolo si costruisce apertamente in opposizione al genere diaristico, sviluppando immaginari specifici dalle evidenti rilevanze testuali e socio-poetiche. 